\title{
Methods for Animal Brain Mapping
}

\author{
Vaclava PIORECKA ${ }^{1,2}$, Vladimir KRAJCA ${ }^{1,2}$, Filip TYLS ${ }^{2,3}$, Tomas PALENICEK ${ }^{2,3}$ \\ ${ }^{1}$ Faculty of Biomedical Engineering, Czech Technical University in Prague, Czech Republic \\ 2 National Institute of Mental Health, Prague, Czech Republic \\ 3 3rd Faculty of Medicine, Charles University in Prague \\ vaclava.piorecka@fbmi.cvut.cz, vaclava.piorecka@nudz.cz \\ Submitted October 25, 2017 / Accepted June 1, 2018
}

\begin{abstract}
Measurements of brain electrical activity in animals are essential for the validation of the pharmaco-effect of drugs. The way to evaluate these recordings should be comparable to that of EEG in humans. Methods that visualize the results of the measured EEG recording include brain mapping in two-dimensional or three-dimensional space. The most commonly used methods of interpolation techniques in humans are spherical splines and $3 D$ splines. We measured nine brains of Wistar rats and compared them with a brain model from the atlas (Brain Atlas Reconstructor, BAR). We validated the brain model of Wistar rat for future use. We implemented a module in MATLAB 2015a for brain mapping, specifically, we implemented algorithms for spherical and $3 D$ spline mapping. The root mean square error of the spherical spline method is 0.5943 in the case of testing signal and $0.6291 / 0.6388$ in the case of real data estimation. The root mean square error of the $3 D$ spline method is 0.4334 in the case of testing signal and 0.0849/0.0768 in the case of real data estimation. Our results show that the $3 D$ spline method with the projection on sphere gives significantly better $3 D$ potential map than spherical splines.
\end{abstract}

\section{Keywords}

Animal EEG, brain mapping, 3D spline, spherical spline

\section{Introduction}

Electroencephalography (EEG) is a commonly used method for studying brain electrical activity. This method is also used to study the effect of drugs on the brain. The animal pharmaco-EEG (p-EEG) is applied for its biomarker potential-extensive databases of p-EEG and polysomnography studies in rats and mice hold EEG signatures of a broad collection of psychoactive reference and test compounds [1]. Therefore, it is important to measure and evaluate human and animal EEG similarly.

EEG measurement of humans is a method that is noninvasive, fast and has a standardized electrode system. In open literature, EEG measurement on animal models has not a standardized system. Moreover, the comonly used system are rather invasive. Different number of electrodes and differ- ent electrode location are used in animal studies. For example 12 electrodes was implanted in the study of Palenicek et al. [2] and Fujakova et al. [3], 7 electrodes were implanted in the study of Cavelli et al. [4], 2-4 electrodes were implanted in the study of Cambiaghi et al. [5] and 32 electrodes were implanted in the study of Bae et al. [6].

We can look for abnormalities in 1D electrical voltage signals measured from the scalp or in 2D or 3D maps as a colorful (intensively) distinct area from their surroundings. The map means a spatial representation of the selected magnitude resulting from electrical activity. The most commonly use methods for brain mapping are: amplitude, frequency, coherence, cordance. [7]

Topographical maps are an excellent and nearly ubiquitously used method for showing the spatial distribution of EEG results [8]. Brain mapping is a convenient method for searching for hidden information, that is not visible from the shape of the EEG signal. Brain mapping methods are used for example in the study of cognitive deterioration in the eldery [9], as a tool to study neurodegeneartion [10] or in the study of microstates dynamics. The microstates dynamics can be used for study of the Alzheimer disease [11], in the study of the schizophrenia [12] or in the study of the depression [13].

There are several types of 3D interpolation methods in human brain mapping. Polynomial based methods and 3D barycentric interpolation methods were used in the study of Soufflet et al. [14]. There is also a technique that can be used for modeling of human head surface on the basis of quadratic triangular B-splines [15]. Perrin at al. suggested methods of spherical spline and 3D spline interpolation [16], [17]. Spherical splines are the most used interpolation methods for brain mapping in humans [18], [19]. However the rats brain can not be approximated well by a simple sphere. Based on results in the studies [18], [19] we can say, that we get better results when we use 3D splines for brain mapping in humans. In this paper, the 3D splines are applied in rats for the first time. Efficiency of interpolation methods is computed by using Root Mean Squared error (RMS) between measured and interpolated signals under the electrodes [18], [19]. First step in brain mapping with spherical splines and 3D splines is the projection on the sphere [18-20]. 
Our major goals are the validation of Wistar rat brain and implementation of three-dimensional interpolation methods for brain mapping in animal models.

\section{Data}

The EEG signals were recorded using BrainScope data acquisition system (Unimedis, Prague, Czech Republic). The bandwidth of this system amplifier was $0.15-70.00 \mathrm{~Hz}$. The system's dynamic range was $\pm 500 \mu \mathrm{V}$. The data were recorded with the sampling rate $250 \mathrm{~Hz}$.

EEG was recorded from 12 electrodes in freely mooving Wistar rats. These electrodes were placed in rat cortex. The EEG was recorded using a BrainScope system 7 days after the implantation of the electrodes. Methods of electrode implantation and recording were in detaile described in [2], [3]. All the experiments respected the Guidelines of the European Union (86/609/EU) and followed the instructions of the National Committee for the Care and Use of Laboratory Animals.

\section{Methods}

\subsection{Brain Model}

We used a brain model from Brain Atlas Reconstructor (BAR) [21], [22] as a template for spline mapping. Morover, the template was validated by measuring the structure of additional nine rat brains.

The brain model is uniquely described by the following parameters: faces, edges and vertices. The faces consist of triangles (triangle mesh) and defined as a closed set of edges. Vertices represent the position of points in space. For our purpose, we defined model just with faces and vertices. From the whole 3D brain surface model we used only the cortex area as a region of interest since the splines can not extrapolate the signals to the deep brain structures.
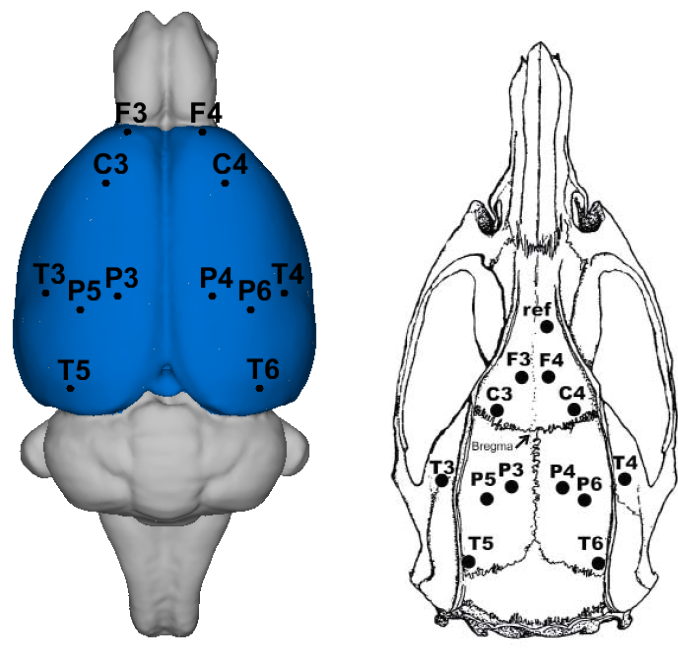

Fig. 1. Location and names of electrodes on rat's brain (left) and skull (right).

\subsection{Animal Brain Mapping}

For animal brain mapping we used two types of spline interpolation: spherical splines and 3D splines. Both of these methods are used in human brain mapping but not applied in rats yet [16-20].

The spline curve computation process consists of two parts. The first part is projection electrodes and model's position of the semi-sphere. In the second part we apply spline interpolation functions, see Fig. 2.

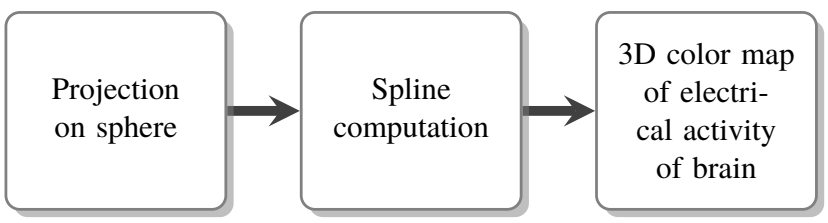

Fig. 2. Algorithm of brain mapping.

\subsubsection{Projection on Sphere}

Projection of real geometry of the rat brain on a sphere is defined by (1). In this equation variables $[x, y, z]$ represent Cartesian coordinates of each point of the brain model, which are projected on a sphere. Variables $\left[x_{0}, y_{0}, z_{0}\right]$ describe the center point of the brain model and $r$ represent the radius of the sphere.

$$
\left(x-x_{0}\right)^{2}+\left(y-y_{0}\right)^{2}+\left(z-z_{0}\right)^{2}=r^{2} .
$$

At first we calculate ideal radius and an ideal center of the sphere. The ideal center and the radius $(r)$ are calculated by minimizing function in (1).

Let the vector $P O S$ be the vector of all model point positions. All points are moved in the coordinate system centered at the center of the sphere $\left(x_{0}, y_{0}, z_{0}\right)$, see (2). Than we compute length of this vector: $|P O S|$, see (3). We scale the vector by using length of ideal radius of the sphere and we get a new vector $Q_{P O S}$, see (4). As a last step we change of the coordinate system to original so we get projection of model point position on the sphere sphPOS, see (5).

$$
\begin{aligned}
P O S\left(x^{\prime}, y^{\prime}, z^{\prime}\right) & =\operatorname{POS}\left(x-x_{0}, y-y_{0}, z-z_{0}\right), \\
|P O S| & =\sqrt{x^{\prime 2}+y^{\prime 2}+z^{\prime 2}} \\
Q_{P O S} & =(r /|P O S|) \cdot P O S, \\
\operatorname{sphPOS} & =Q_{P O S}+\left(x_{0}, y_{0}, z_{0}\right) .
\end{aligned}
$$

This algorithm is also used for projection of electrode positions on the sphere. For this projection we use the same center and radius as the ideal center and the ideal radius of model point positions. 


\subsubsection{Spherical Splines}

Brain mapping through spherical splines can be divided into two parts. In the first part we calculate the coefficients of the electrodes $C$ and the coefficients of the model points $G x$. In the second part we calculate the intensity at all points of the model. Spherical splines use Legendre polynomials. Legendre polynomials of order $n$ form a complete set of base functions in the range of $-1<x<1$ on the spherical surface. Legendre polynomial $\mathrm{n}$-th degree can be expressed using the Rodrigues formula. The value of $x$ in $P_{n}(x)$ represents the angle between the position of the electrode $\vec{r}_{i}$ and the interpolated point $\vec{r}$ [16], [17].

\begin{tabular}{ll}
\hline Variable & \multicolumn{1}{c}{ Description } \\
\hline \hline$N$ & number of electrodes \\
$M$ & number of points on brain model \\
$g_{\mathrm{m}}$ & Euclidean distance in 3D space \\
$G x(i, j)$ & matrix of coefficients of brain model, size $[N, M]$ \\
$C$ & matrix of electrodes coefficients, size $[N, N]$ \\
$V$ & vector of actual EEG values, size $[N, 1]$ \\
$U$ & intensity in each point of model, size is $[M, 1]$ \\
\hline
\end{tabular}

Tab. 1. Variables.

The first part of the coefficient calculation is more timeconsuming. If we do not change the brain model or the position of the electrodes then this part is calculated only once.

The algorithm describing the calculation of model point coefficients is illustrated in the pseudocode, see Algorithm 1 and 2. The first step is to calculate the Euclidean distance between the individual electrodes. We use this distance for computation of Legendre polynomials. In our case we chose the Legendre polynomial order $n=4$ and we compute the first 7 members of the polynomial. These parameters are chosed with respect to parameter settings for human brain mapping [17].

$$
P_{n}(x)=\left(2^{n} n !\right)^{-1} \frac{\mathrm{d}^{n}}{\mathrm{~d} x^{n}}\left[\left(x^{2}-1\right)^{n}\right]
$$

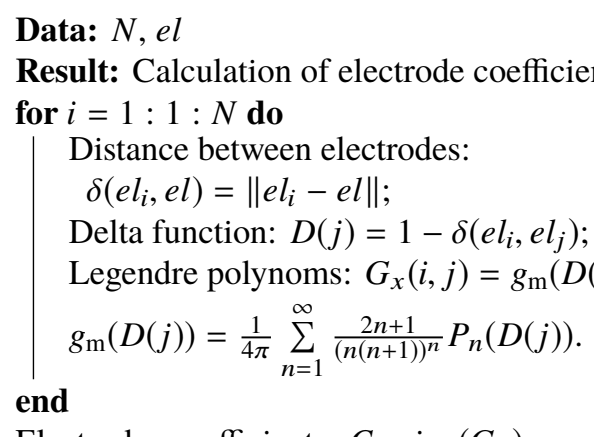

Electrodes coefficients: $C=\operatorname{inv}\left(G_{x}\right)$;

Algorithm 1: Calculation of the electrode coefficients. Output is the matrix with size $[N, N]$, where $N$ is the number of electrodes and $e l$ is the matrix of the electrode positions.
We calculate the coefficients of the model points based on the same algorithm as in the calculation of the electrode coefficients. The electrode coefficients are defined by the variable $G_{x}(i, j)$. Quantification of coefficients is the slowest operation that depends on the number of contour models. The more accurate the model, the longer it takes to computation of the model point coefficients. Algorithm 2 describes the pseudocode of the algorithm for calculating model point coefficients.

Data: $M, p n t, e l$

Result: Calculation of Model Point Coefficients

for $i=1: 1: M$ do

Distance between points and electrodes:

$\delta\left(p n t_{i}, e l\right)=\left\|p n t_{i}-e l\right\|$

Determining values using the delta function:

$D(j)=1-\delta\left(p n t_{i}, e l\right)$;

Calculate the model point matrix:

$G x(i, j)=g_{\mathrm{m}}(D(j))$;

$g_{m}(D(j))=\frac{1}{4 \pi} \sum_{n=1}^{\infty} \frac{2 n+1}{(n(n+1))^{n}} P_{n}(D(j))$

end

Algorithm 2: Calculation of the model point coefficients. Output is the matrix $G x$ with size $[M, N]$, where $M$ is the number of points of brain model, $N$ is the number of electrodes, pnt is the matrix of the model point positions and $e l$ is the matrix of the electrode positions.

When we know the coefficients of model points and the coefficients of the electrodes we can compute intensity in all points of the model. Let $V$ be the vector of measured values under the electrodes, $C$ be the matrix of electrode coefficients and $G x$ be the matrix of model point coefficients, then $U(i)$ is the vector of intensity at each point of the brain model.

$$
\begin{aligned}
C^{\prime} & =C \times V, \\
U(i) & =\sum_{j=1}^{M} C^{\prime}(j, 1) \cdot G x(i, j) .
\end{aligned}
$$

\subsubsection{D Splines}

The three-dimensional splines were originally introduced by Perrin et al. [16] in two-dimensional brain mapping. Then the 3D splines were implemented in three-dimensional brain mapping in several studies $[17,20,23,24]$. The simplified scheme of the $3 \mathrm{D}$ spline algorithm is described in Fig. 3.

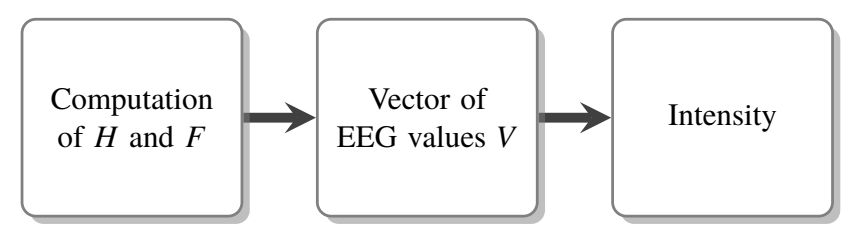

Fig. 3. Simplified scheme of 3D spline algorithm. 
Let $[x, y, z]$ be a Cartesian coordinates of brain model, $\left[x_{\mathrm{e}}, y_{\mathrm{e}}, z_{\mathrm{e}}\right]$ be a Cartesian coordinates of electrode positions and $m$ be an order of interpolation. Intensity at each point of brain model is defined by:

$$
\begin{array}{r}
U_{m}(x, y, z)=\sum_{i=1}^{N} p_{i} \cdot H_{m}+\sum_{d=0}^{m-1} \sum_{k=0}^{d} \sum_{g=0}^{k} q_{d k g} \cdot F_{m}, \\
F_{m}=\sum_{d=0}^{m-1} \sum_{k=0}^{d} \sum_{g=0}^{k} q_{d k g} \cdot x^{d-k} \cdot y^{k-g} \cdot z^{g}, \\
H_{m}=\left(\left(x-x_{\mathrm{e}}\right)^{2}+\left(y-y_{\mathrm{e}}\right)^{2}+\left(z-z_{\mathrm{e}}\right)^{2}\right)^{\frac{(2 m-3)}{2}} .
\end{array}
$$

The intensity at each point of the brain model $U_{m}$ depends on $N$ coefficients $p_{i}$, where $N$ represents a number of electrodes. These coefficients are represented in $P$ vector. The intensity depends also on $[m \cdot(m+1) \cdot(m+2) / 6]$ coefficients $q_{d k g}$ which are represented in $Q$ vector. Vectors $P$ and $Q$ are obtained by solving the following matrix system:

$$
\left[\begin{array}{ll}
H & F \\
F^{t} & 0
\end{array}\right] \cdot\left[\begin{array}{l}
P \\
Q
\end{array}\right]=\left[\begin{array}{l}
V \\
0
\end{array}\right]
$$

$V$ is the vector of intensity under all of the electrodes, $V=\left[V_{1}, V_{2}, \ldots, V_{N}\right]$. In this system matrix $H$ represents distances between electrode positions, depending on the order of interpolation $m$.

$$
H(i, j)=\left(\left(x_{\mathrm{e} i}-x_{\mathrm{e} j}\right)^{2}+\left(y_{\mathrm{e} i}-y_{\mathrm{e} j}\right)^{2}+\left(z_{\mathrm{e} i}-z_{\mathrm{e} j}\right)^{2}\right)^{\frac{(2 m-3)}{2}} .
$$

The matrix $F$ represents the relationship between the Cartesian coordinates of each electrode, depending on the order of interpolation $m$.

$$
F=\sum_{d=0}^{m-1} \sum_{k=0}^{d} \sum_{g=0}^{k} q_{d k g} \cdot x^{d-k} \cdot y^{k-g} \cdot z^{g}
$$

Solution of the system of equations (12) is a vector $[P, Q]$. If these vectors are determined, we can use (9) to calculate the intensity at each point of the brain model.

Based on previous research, we chose interpolation order $m=3$ [18]. In this case each row of matrix $F$ is defined as:

$$
F_{i}=\left[1, x_{i}, y_{i}, z_{i}, x_{i}^{2}, x_{i} \cdot y_{i}, z_{i} \cdot x_{i}, y_{i}^{2}, z_{i} \cdot y_{i}, z_{i}^{2}\right]
$$

where $i$ is the number of electrodes.

\section{Results}

\subsection{Model Validation}

We compared the scan of the rat brain from the atlas [21], [22] and the scanned rat brains. Rat brains were aligned to the atlas according to the smallest deviation. The RMS error of alignment was $0.695 \mathrm{~mm}$ and average error of alignment was $0.344 \mathrm{~mm}$, see Tab. 2 .

We computed the absolute difference in $\mathrm{mm}$ between the model from BAR atlas and the average of the scanned brains. The number of points with different positions are described in Tab. 3 and in Fig. 4.

\begin{tabular}{lc}
\hline \multicolumn{1}{c}{ Variable } & Deviation $(\mathbf{m m})$ \\
\hline \hline Max. upper deviation & 1.4463 \\
Max. lower deviation & -1.6596 \\
Average upper deviation & 0.0911 \\
Average lower deviation & -0.3000 \\
Standard deviation & 0.2388 \\
\hline
\end{tabular}

Tab. 2. This table describes maximal upper and lower deviation the between the average of scanned brains and model of brain from BAR - [21], [22].

\begin{tabular}{cccc}
\hline \multicolumn{2}{c}{$\begin{array}{c}\text { Deviation interval } \\
\min (\mathrm{mm})\end{array}$} & $\begin{array}{c}\text { No. points } \\
(-)\end{array}$ & $\begin{array}{c}\text { Percentage } \\
(\%)\end{array}$ \\
\hline \hline-1.000 & -0.900 & 469 & $0.786 \%$ \\
-0.900 & -0.800 & 840 & $1.407 \%$ \\
-0.800 & -0.700 & 1676 & $2.808 \%$ \\
-0.700 & -0.600 & 2254 & $3.777 \%$ \\
-0.600 & -0.500 & 2772 & $4.645 \%$ \\
-0.500 & -0.400 & 4494 & $7.530 \%$ \\
-0.400 & -0.300 & 8286 & $13.884 \%$ \\
-0.300 & -0.200 & 10966 & $18.374 \%$ \\
-0.200 & -0.100 & 11178 & $18.729 \%$ \\
-0.100 & 0.100 & 13413 & $22.474 \%$ \\
0.100 & 0.200 & 2508 & $4.202 \%$ \\
0.200 & 0.300 & 353 & $0.591 \%$ \\
0.300 & 0.400 & 236 & $0.395 \%$ \\
0.400 & 0.500 & 81 & $0.136 \%$ \\
0.500 & 0.600 & 16 & $0.027 \%$ \\
0.600 & 0.700 & 15 & $0.025 \%$ \\
0.700 & 0.800 & 1 & $0.002 \%$ \\
0.800 & 0.900 & 2 & $0.003 \%$ \\
0.900 & 1.000 & 1 & $0.002 \%$ \\
\hline
\end{tabular}

Tab. 3. This table represents the deviation distribution between the average of scanned brains and model of brain from BAR - [21], [22].

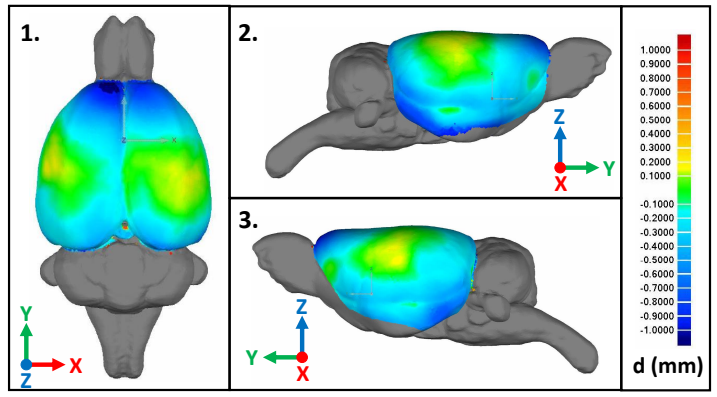

Fig. 4. This figure describes deviation distribution between average of all brains models and model of the brain from BAR - [21], [22]. Created by: Jiří Petr, SolidVision, s.r.o. 


\begin{tabular}{lccc}
\hline & \multicolumn{3}{c}{ Root mean square error (RMS), (-) } \\
& Behavioral active & Behavioral passive & Test signal \\
\hline \hline 3D spline & 0.0359 & 0.0353 & 0.0694 \\
Sphere spline & 0.6291 & 0.6388 & 0.5934 \\
3D spline, non sphere & 0.0849 & 0.0768 & 0.4334 \\
\hline
\end{tabular}

Tab. 4. Evaluation of interpolation methods.

\subsection{Interpolation Methods}

We evaluated the findings based on the calculation of the normalized Root Mean Squared error (RMS). This parameter finds differences between measured value on each electrode $U_{\text {real }}(n)$ and the interpolated value of each electrode $U_{\text {interpolated }}(n)$.

$$
R M S=\frac{\left\|U_{\text {real }}(n)-U_{\text {interpolated }}(n)\right\|}{\left\|U_{\text {real }}(n)\right\|} .
$$

In our case we found four nearest points of brain model to each electrode location. We computed the interpolated value as a mean of the potential of this four nearest points. We compared test signal, behavioral active data and behavioral passive data.

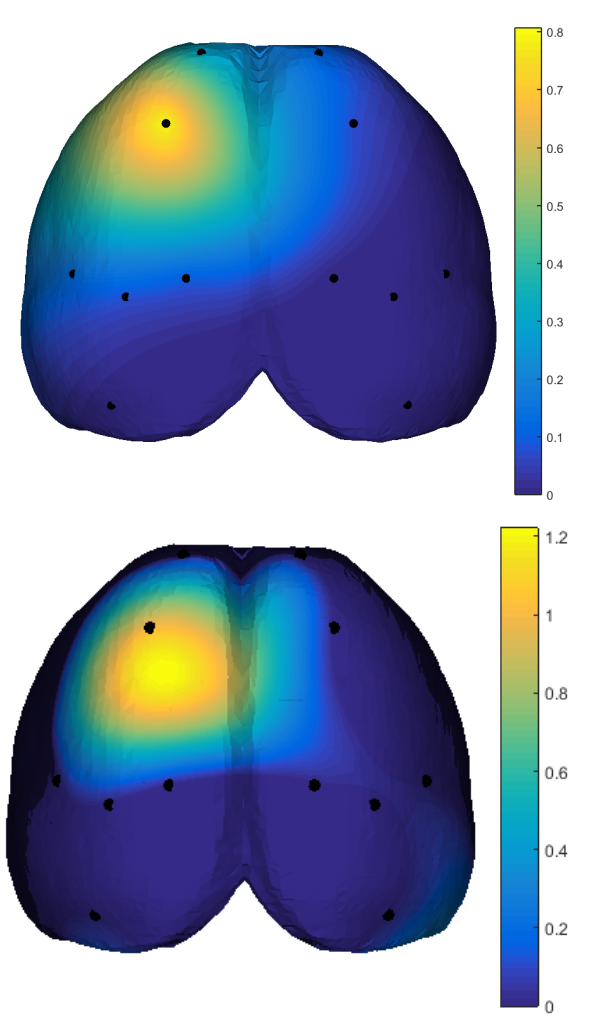

Fig. 5. Figure on the left shows the results after 3D spline mapping. The picture on the right show the results after the spherical spline mapping. Both figures represent response to testing signal: $V=[0,0,1,0,0,0,0,0,0,0,0,0]$.
The test data are vectors, where voltage under one electrode is $1 \mu \mathrm{V}$ and below the other electrodes there is a zero intensity value. We calculated the RMS error values for the test data in which we have activated all of the electrodes. The RMS error value was calculated as the average of all RMS errors in the test data.

Behavioral active and behavioral passive data were measured on 9 Wistar rats. Data was transformed by Fast Fourier Transform and spectral analyses were performed by absolute spectrum computation using Welch method of modified periodograms [25].

There are examples of two interpolation methods in Fig. 5. The left one represents spherical spline interpolation on test signal. The maximum of intensity is reflected around the electrode with a non-zero signal. The signal is spreading towards the other electrodes for which the signal should be zero. This also corresponds to the RMS error results in Tab. 4. The right part of Fig. 5 represents 3D spline interpolation on test signal. The maximum of intensity is shifted and is not directly below the electrode with non-zero signal. Below the electrode, the value is close to 1 , which approximates the value of the input signal. We can also notice that the signal does not interfere with other electrodes. The RMS error in case of using 3D spline interpolation is lower than spherical spline interpolation, see Tab. 4.

\section{Conclusion}

Measurements of brain electrical activity in animals are essential for the validation of drug effect on the organism. The way to evaluate these recordings should be comparable to that of EEG on humans. The open source software was developed for animal and human 3D brain mapping. This software was implemented in MATLAB 2015a.

We validated the brain model for future use in our laboratory. By validating the model, we obtained the following statistical values: maximal upper deviation $1.466 \mathrm{~mm}$, maximal lower deviation $-1.660 \mathrm{~mm}$, average deviation $0.091 / 0.300 \mathrm{~mm}$, standard deviation $0.239 \mathrm{~mm}$.

We implemented spherical splines and 3D spline method for 3D brain mapping in rats. We computed RMS error of three types of EEG data - test signal, behavioral active and behavioral passive data. Our results showed that the 3D spline interpolation method with projection on sphere gives significantly better 3D potential map than spherical spline interpolation method. 


\section{Acknowledgments}

This work was supported by the Grant Agency of the Czech Technical University in Prague, grant number SGS18/158/OHK4/2T/17 with topic: The topographic brain mapping, by the Grant Agency of Czech Republic with topic: Temporal context in analysis of long-term non-stationary multidimensional signal, register number $17-20480 \mathrm{~S}$ and by the project No. LO1611 from the MEYS under the NPU I program, PROGRES Q35 and by MH CZ - DRO (National Institute of Mental Health-NIMH) project No. 00023752.

\section{References}

[1] DRINKENBURG, W. H. I. M. Encyclopedia of Psychopharmacology. 2nd ed. Berlin: Springer, 2015. ISBN: 978-3-642-27772-6

[2] PÁlENÍČEK, T., FUJÁKOVÁ, M., BRUNOVSKÝ, M., et al. Behavioral, neurochemical and pharmaco-EEG profiles of the psychedelic drug 4-bromo-2,5-dimethoxyphenethylamine (2C-B) in rats. Psychopharmacology, 2013, vol. 225, no. 1, p. 75-93. DOI: $10.1007 / \mathrm{s} 00213-012-2797-7$

[3] FUJÁKOVÁ, M., PÁLENÍČEK, T., BRUNOVSKÝ, M., et al. The effect of ((-)-2-oxa-4-aminobicyclo[3.1.0]hexane-2,6-dicarboxylic acid (LY379268), an mGlu2/3 receptor agonist, on EEG power spectra and coherence in ketamine model of psychosis. Pharmacology Biochemistry and Behavior, 2014, vol. 122, p. 212-221. DOI: $10.1016 /$ j.pbb.2014.03.001

[4] CAVELli, M., CASTRO, S., SCHWARZKOPF, N., et al. Coherent neocortical gamma oscillations decrease during REM sleep in the rat. Behavioural Brain Research, 2015, vol. 281, p. 318-325. DOI: $10.1016 /$ j.bbr.2014.12.050

[5] CAMBIAGHI, M., MAGRI, L., CURSI, M. Importance of EEG in validating the chronic effects of drugs: Suggestions from animal models of epilepsy treated with rapamycin. Seizure, 2015, vol. 27, p. 30-39. DOI: 10.1016/j.seizure.2015.02.015

[6] BAE, J., DESHMUKH, A., SONG Y., RIERA, J. Brain source imaging in preclinical rat models of focal epilepsy using high-resolution EEG recordings. Journal of Visualized Experiments, 2015, vol. 100, p. 1-12. DOI: $10.3791 / 52700$

[7] SANEI S., CHAMBeRS J. A. EEG Signal Processing. 1st ed. Southern Gate, Chichester (England): John Wiley \& Sons, 2007. ISBN: 978-0-470-02581-9

[8] COHEN, M. X. Analyzing Neural Time Series Data: Theory and Practice. The MIT Press, 2014. ISBN: 0262019876

[9] PRICHEP, L. S., JOHN, E. R., FERRIS, S. H., et al. Quantitative EEG correlates of cognitive deterioration in the elderly. Neurobiology of Aging, 1994, vol. 15, no. 1, p. 85-90. DOI: 10.1016/01974580(94)90147-3

[10] APOSTOLOVA, L. G., THOMSPON, P. M. Brain mapping as a tool to study neurodegeneration. Neurotherapeutics, 2007, vol. 4, p. 387-400. DOI: 10.1016/j.nurt.2007.05.009

[11] STEVENS, A., KIRCHER, T. Cognitive decline unlike normal aging is associated with alterations of EEG temporo-spatial characteristics. European Archives of Psychiatry and Clinical Neuroscience, 1998, vol. 248, no. 5, p. 259-266. DOI: 10.1007/s004060050047
[12] ANDREOU, CH., FABER, P. L., LEICHT, G., et al. Resting-state connectivity in the prodromal phase of schizophrenia: Insights from EEG microstates. Schizophrenia Research, 2014, vol. 152, no. 2-3, p. 513-520. DOI: 10.1016/j.schres.2013.12.008

[13] STRIK, W. K., CHIARAMONTI, R., MUSCAS, G. C., et al. Decreased EEG microstate duration and anteriorisation of the brain electrical fields in mild and moderate dementia of the Alzheimer type. Psychiatry Research: Neuroimaging, 1997, vol. 75, no. 3, p. 183-191. DOI: $10.1016 / \mathrm{S} 0925-4927(97) 00054-1$

[14] SOUFFLET, L., TOUSSAINT, M., LUTHRINGER, R., et al. A statistical evaluation of the main interpolation methods applied to 3-dimensional EEG mapping. Electroencephalography and Clinical Neurophysiology, 1991, vol. 79, no. 5, p. 393-402. DOI: 10.1016/0013-4694(91)90204-H

[15] MIHALIK, J. Modeling of human head surface by using triangular B-splines. Radioengineering, 2010, vol. 19, no. 1, p. 39-45. DOI: $10.13164 / \mathrm{re}$

[16] PERRIN, F., PERNIER, J., BERTRAND, O., et al. Mapping of scalp potentials by surface spline interpolation. Electroencephalography and Clinical Neurophysiology, 1987, vol. 66, no. 1, p. 75-81. DOI: 10.1016/0013-4694(87)90141-6

[17] PERRIN, F., PERNIER, J., BERTRAND, O., ECHALliER, J. F. Spherical splines for scalp potential and current density mapping. Electroencephalography and Clinical Neurophysiology, 1989, vol. 72, no. 2, p. 184-187. DOI: 10.1016/0013-4694(89)90180-6

[18] NOUIRA, I., ABDALLAH, A. B., BEDOU, M. H. EEG potential mapping by 3D interpolation methods. In Proceedings of the International Conference on Multimedia Computing and Systems (ICMCS). Marrakech (Morocco), 2014, p. 469-474. DOI: 10.1109/ICMCS.2014.6911297

[19] NOUIRA, I., ABDALLAH, A. B., BEDOU, M. H. Three-dimensional interpolation methods to spatiotemporal EEG mapping during various behavioral states. Signal, Image and Video Processing, 2016, vol. 10, no. 5, p. 943-949. DOI: 10.1007/s11760-015-0844-7

[20] FERREE, T. C., Electrical Geodesics, Inc. Spline Interpolation of the Scalp EEG. Technical Note, 6 pages, [Online] Cited 2017-10-12. Avaible at: https://www.egi.com/images/kb/SplineInterpolation.pdf

[21] MAJKA, P., KOWALSKI, J. M., CHLODZINSKA, N., WÓJCIK, D. 3D brain atlas reconstructor service online repository of threedimensional models of brain structures. Neuroinformatics, 2013, vol. 11, no. 4, p. 507-518. DOI: 10.1007/s12021-013-9199-9

[22] CALABRESE, E., BADEA, A., WATSON, C., et al. A quantitative magnetic resonance histology atlas of postnatal rat brain development with regional estimates of growth and variability. NeuroImage, 2013, vol. 71, p. 196-206. DOI: 10.1016/j.neuroimage.2013.01.017

[23] ABDOUN, O., JOUCLA, S., MAZZOCCO, C., YVERT, B. NeuroMap: A spline-based interactive open-source software for spatiotemporal mapping of 2D and 3D MEA data. Frontiers in Neuroinformatics, 2011, vol. 4, p. 1-9. DOI: 10.3389/fninf.2010.00119

[24] LAW, S. K., NUNEZ, P. L., WIJESINGHE, R. S. High-resolution EEG using spline generated surface Laplacians on spherical and ellipsoidal surfaces. IEEE Transactions on Bio-Medical Engineering, 1993, vol. 40, no. 2, p. 145-153. DOI: 10.1109/10.212068

[25] WELCH, P. The use of fast Fourier transform for the estimation of power spectra: A method based on time averaging over short, modified periodograms. IEEE Transactions on Audio and Electroacoustics, 1967, vol. 15, no. 2, p. 70-73. DOI: 10.1109/TAU.1967.1161901 


\section{About the Authors...}

Vaclava PIORECKA (corresponding author) currently attends doctoral study at the Faculty of Biomedical Engineering of the Czech Technical University in Prague. Her research interests include bio-signal processing, brain-mapping and EEG graphoelements detection and classification.

Vladimir KRAJCA was born in 1955. He received his Ph.D. and M.Sc. at the Czech Technical University in Prague in 1979, respectively 1985 . Currently he has the position of Associated Professor at the Faculty of Biomedical Engineering, Czech Technical University in Prague. His research interests include digital signal processing, multichannel adaptive segmentation, cluster analysis, artificial intelligence, neural networks, genetic algorithms, long-term monitoring, analysis of epilepsy and other brain disorders. He has sixty nine contributions, that are indexed in the Web of Science; his $\mathrm{h}$-index is 11 .

Filip TYLS (1985, Prague, Czech Republic) is a psychiatrist and neuroscientist with main interest in translational models of acute psychosis and psychedelic substances. He completed 1st Medical Faculty and Ph.D. program Neurosciences on 3rd Medical Faculty on Charles University in Prague. He recieved a specialization in electroencephalography and is in training of Gestalt psychotherapy. He has experiences as psychedelic sitter in a team working on pilot clinical trial with psilocybin in the Czech Republic. He has published several papers in local as well as foregin journals (e.g. review Psilocybin - summary of knowledge).

Tomas PALENICEK started his career as a Ph.D. student in preclinical research, studying the neurobiology of schizophrenia in animal models. Shortly after, he received his first grant funding and extended his research interests towards the neurobiology of psychedelic drugs (e.g., LSD, psilocin, mescaline, 2C-B), entactogens (ecstasy / MDMA) and new synthetic drugs. Simultaneous to working on his Ph.D. he passed his training as a clinical psychiatrist with a specialism in clinical electroencephalography. He defended his thesis in early 2009 and in 2012 he became a licensed psychiatrist. Over the last few years he contributes significantly to human clinical research. He is involved as a co-investigator of ketamine projects in NIHM CR, where ketamine is used to model psychosis and to treat depression in humans. For the last four years he is a principle investigator of the first project in the Czech Republic that is intended to study the acute effects of cannabis in healthy volunteers. Finally, he is also a principle investigator of the first human clinical trial in the Czech Republic studying the effects of psilocybin in human volunteers. Currently his research interests are oriented to the area of EEG functional connectivity. He continues his clinical practice as a psychiatrist in local outpatient clinic. 